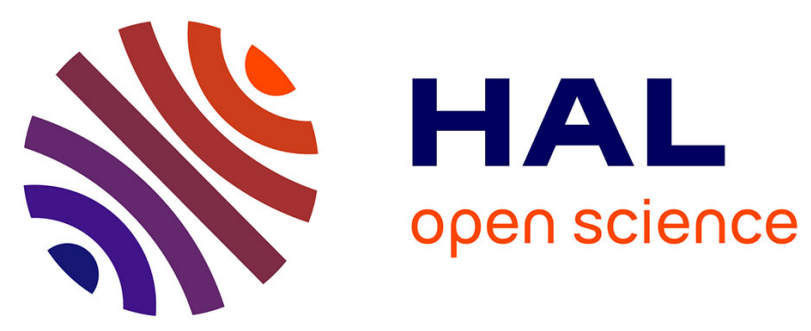

\title{
Recursive hybrid CRB for Markovian systems with time-variant measurement parameters
}

Jérôme Galy, Alexandre Renaux, Eric Chaumette, François Vincent, Pascal

Larzabal

\section{- To cite this version:}

Jérôme Galy, Alexandre Renaux, Eric Chaumette, François Vincent, Pascal Larzabal. Recursive hybrid CRB for Markovian systems with time-variant measurement parameters. CAMSAP: Computational Advances in Multi-Sensor Adaptive Processing, Dec 2015, Cancun, Mexico. pp.473-476, 10.1109/CAMSAP.2015.7383839 . hal-01447112

\section{HAL Id: hal-01447112 \\ https://hal.science/hal-01447112}

Submitted on 26 Jan 2017

HAL is a multi-disciplinary open access archive for the deposit and dissemination of scientific research documents, whether they are published or not. The documents may come from teaching and research institutions in France or abroad, or from public or private research centers.
L'archive ouverte pluridisciplinaire HAL, est destinée au dépôt et à la diffusion de documents scientifiques de niveau recherche, publiés ou non, émanant des établissements d'enseignement et de recherche français ou étrangers, des laboratoires publics ou privés. 


\section{Open Archive TOULOUSE Archive Ouverte (OATAO)}

OATAO is an open access repository that collects the work of Toulouse researchers and makes it freely available over the web where possible.

This is an author-deposited version published in: http://oatao.univ-toulouse.fr/ Eprints ID: 16660

To cite this version: Galy, Jerome and Renaux, Alexandre and Chaumette, Eric and Vincent, François and Larzabal, Pascal Recursive hybrid CRB for Markovian systems with time-variant measurement parameters. (2016) In: CAMSAP 2015, 13 December 2015 - 16 December 2015 (Cancun, Mexico).

Official URL: http://dx.doi.org/10.1109/CAMSAP.2015.7383839

Any correspondence concerning this service should be sent to the repository administrator: staff-oatao@listes-diff.inp-toulouse.fr 


\title{
Recursive Hybrid CRB for Markovian Systems with Time-Variant Measurement Parameters
}

\author{
Jerome Galy ${ }^{(1)}$, Alexandre Renaux ${ }^{(2)}$, Eric Chaumette ${ }^{(3)}$, Francois Vincent ${ }^{(3)}$, Pascal Larzabal ${ }^{(4)}$ \\ (1) Université de Montpellier 2/LIRMM, 161 rue Ada, Montpellier, France (galy@lirmm.fr) \\ (2) Université Paris-Sud/LSS, 3 Rue Joliot-Curie, Gif-sur-Yvette, France (renaux@1ss.supelec.fr) \\ (3) ISAE-SUPAERO, Université de Toulouse, 31055 Toulouse Cedex 4, France \\ (eric.chaumette@isae.fr, francois.vincent@isae.fr) \\ (4) Université Paris-Sud/SATIE, 61 av. du President Wilson, Cachan, France (pascal.larzabal@satie.ens-cachan.fr)
}

\begin{abstract}
In statistical signal processing, hybrid parameter estimation refers to the case where the parameters vector to estimate contains both deterministic and random parameters. Lately computationally tractable hybrid Cramér-Rao lower bounds for discrete-time Markovian dynamic systems depending on unknown time invariant deterministic parameters has been released. However in many applications (radar, sonar, telecoms, ...) the unknown deterministic parameters of the measurement model are time variant which prevents from using the aforementioned bounds. It is therefore the aim of this communication to tackle this issue by introducing new computationally tractable hybrid Cramér-Rao lower bounds.
\end{abstract}

\section{INTRODUCTION}

In the Bayesian estimation framework, discrete-time Markovian dynamic systems (MDS) arises in various applications such as adaptive control, analysis and prediction of nonstationary time series [1], or signal source tracking (radar, sonar, telecoms) [2]. As is well known, the optimal estimator for this problem cannot be built in general, and it is necessary to turn to one of the large number of existing suboptimal filtering techniques [1][2]. Assessing the achievable performance may be difficult, and we have to resort to simulations and comparing proximity to Bayesian lower bounds corresponding to optimum performance [2]. Actually, most discrete-time MDS incorporate some deterministic parameters which can be either known or unknown according to the experimental conditions [2]. Even when the deterministic parameters are known, some of the true values may originate from a prior calibration process which accuracy impacts on the optimum performance of random parameter estimates. In both cases, there is a need for computationally tractable hybrid lower bounds for discretetime MDS depending on unknown deterministic parameters.

The first hybrid lower bound, the so-called hybrid CramérRao bound (HCRB), has been introduced in [3], extended in [4] to the case where the prior probability density function (pdf) of the random parameters depends on deterministic parameters, and its asymptotic tightness has been further analyzed in [5]. This characterization of hybrid estimation has

This work has been partially supported by the European Network of excellence NEWCOM\#, by the iCODE institute, research project of the IDEX Paris-Saclay, by the DGA/DGCIS, by the DGA/MRIS (2015.60.0090.00.470.75.01) and by the Display-Mastodons project of CNRS. been generalized by Reuven and Messer [6] who introduced the first "large-error" hybrid bound, the so-called hybrid Barankin Bound (HBB), in order to handle the threshold phenomena and of which one limiting form yields the HCRB. This seminal work [6] has been lately extended to new "large-error" hybrid bounds [7][8][9] in order to improve the estimation of the transition region where the threshold phenomena occurs. Unfortunately, the computational cost of hybrid "large-error" bounds is prohibitive in most applications when the number of unknown parameters increases. All these works have shown that, like the deterministic CRB and Bayesian CRB (BCRB), the HCRB is valid in the asymptotic region only, i.e., when signal to noise ratio is high or the number of observations (measurements) is large.

Therefore, provided that one keeps in mind its limitations, the HCRB is a lower bound of great interest for system analysis and design in the asymptotic region. For that purpose, computationally tractable HCRB for discrete-time MDS depending on unknown time invariant deterministic parameters has been released only lately in [10] and extended in [11]. However in many applications (radar, sonar, telecoms, ...) the unknown deterministic parameters of the measurement model are time variant which prevents from using the bounds derived in [11]. It is therefore the aim of this communication to tackle this issue by introducing new computationally tractable HCRB.

\section{BACKGROUND ON HCRB FOR MDS WITH UNKNOWN DETERMINISTIC PARAMETERS}

In hybrid parameter estimation one wishes to estimate an unknown hybrid parameter vector $(\mathbf{x} ; \boldsymbol{\theta})$ from a random observation vector $\mathbf{y} \in \mathbb{R}^{N^{\prime}}$, where for $L$ column vectors $\mathbf{a}_{l},\left(\mathbf{a}_{1} ; \mathbf{a}_{2} ; \ldots ; \mathbf{a}_{L}\right) \triangleq\left(\mathbf{a}_{1}^{T}, \mathbf{a}_{2}^{T}, \ldots, \mathbf{a}_{L}^{T}\right)^{T}$ denotes the vertical concatenation. Some prior knowledge is available on random parameter $\mathbf{x} \in \mathbb{R}^{P^{\prime}}$ that is incorporated by an a priori pdf $p(\mathbf{x})$ which support is a subset $\Pi_{r}$ of $\mathbb{R}^{P^{\prime}}$. No such knowledge is available on $\boldsymbol{\theta} \in \Pi_{d} \subset \mathbb{R}^{D^{\prime}}$ and thus it is considered deterministic. In the general case, $p(\mathbf{x})$ may depend on the unknown parameter $\boldsymbol{\theta}$, and it is denoted $p(\mathbf{x} \mid \boldsymbol{\theta})$. The conditional pdf of $\mathbf{y}$ given $\mathbf{x}$ parameterized by $\boldsymbol{\theta}$ is $p(\mathbf{y} \mid \mathbf{x}, \boldsymbol{\theta})$ and their joint pdf parameterized by $\boldsymbol{\theta}$ is given by 
$p(\mathbf{y}, \mathbf{x} \mid \boldsymbol{\theta})=p(\mathbf{y} \mid \mathbf{x}, \boldsymbol{\theta}) p(\mathbf{x} \mid \boldsymbol{\theta})$. Then, for any estimators $\widehat{\boldsymbol{\theta}}(\mathbf{y})$ of $\boldsymbol{\theta}$ and $\widehat{\mathbf{x}}(\mathbf{y})$ of $\mathbf{x}$, the HCRB is given by [3][4][5][11]:

$$
\begin{gathered}
E_{\mathbf{y}, \mathbf{x} \mid \boldsymbol{\theta}}\left[\mathbf{e}(\mathbf{y}) \mathbf{e}(\mathbf{y})^{T}\right] \succeq \mathbf{H C R B}_{(\mathbf{x} ; \boldsymbol{\theta})}=\mathbf{J}_{(\mathbf{x} ; \boldsymbol{\theta})}^{-1}, \\
\mathbf{J}_{(\mathbf{x} ; \boldsymbol{\theta})}=E_{\mathbf{y}, \mathbf{x} \mid \boldsymbol{\theta}}\left[\frac{\partial \ln p(\mathbf{y}, \mathbf{x} \mid \boldsymbol{\theta})}{\partial(\mathbf{x} ; \boldsymbol{\theta})} \frac{\partial \ln p(\mathbf{y}, \mathbf{x} \mid \boldsymbol{\theta})}{\partial(\mathbf{x} ; \boldsymbol{\theta})^{T}}\right],
\end{gathered}
$$

where $\mathbf{e}(\mathbf{y})=(\widehat{\mathbf{x}}(\mathbf{y})-\mathbf{x} ; \widehat{\boldsymbol{\theta}}(\mathbf{y})-\boldsymbol{\theta}), E_{\mathbf{y}, \mathbf{x} \mid \boldsymbol{\theta}}[\mathbf{g}(\mathbf{y}, \mathbf{x})]$ is the statistical expectation of the vector of functions $\mathrm{g}()$ with respect to $\mathbf{y}$ and $\mathbf{x}$ parameterized by $\boldsymbol{\theta}$, and for two matrices, $\mathbf{A} \succeq \mathbf{B}$ means that $\mathbf{A}-\mathbf{B}$ is positive semi-definite. The regularity conditions for the hybrid Fisher information matrix (HFIM) $\mathbf{J}_{(\mathbf{x} ; \boldsymbol{\theta})}$ to be of the usual form (2) are [11]: (R1): $\Pi_{r}=\mathbb{R}^{P^{\prime}}$,

(R2): $E_{\mathbf{y}, \mathbf{x} \mid \boldsymbol{\theta}}\left[\frac{\partial \ln p(\mathbf{y}, \mathbf{x} \mid \boldsymbol{\theta})}{\partial x_{p^{\prime}}}{ }^{2}\right], E_{\mathbf{y}, \mathbf{x} \mid \boldsymbol{\theta}}\left[\frac{\partial \ln p(\mathbf{y}, \mathbf{x} \mid \boldsymbol{\theta})}{\partial \theta_{d^{\prime}}}\right]<\infty$.

Moreover, under its usual form (1), the HCRB is a lower bound for the class of estimates satisfying (R3):

$E_{\mathbf{y}, \mathbf{x} \mid \boldsymbol{\theta}}[(\widehat{\mathbf{x}}(\mathbf{y})-\mathbf{x} ; \widehat{\boldsymbol{\theta}}(\mathbf{y})-\boldsymbol{\theta})]=(\boldsymbol{\mu} ; \mathbf{0})$.

Our main concern is the derivation of a computationally tractable HFIM (2) for hybrid discrete-time MDS represented with the state and measurement equations:

$$
\mathbf{x}_{k}=\mathbf{f}_{k-1}\left(\mathbf{x}_{k-1}, \mathbf{w}_{k-1}, \boldsymbol{\alpha}\right), \mathbf{y}_{k}=\mathbf{h}_{k}\left(\mathbf{x}_{k}, \mathbf{v}_{k}, \boldsymbol{\lambda}_{k}\right)
$$

where $k \geq 1$ is a time index, $\mathbf{x}_{k}$ is the $P$-dimensional state vector, $\mathbf{y}_{k}$ is the $N$-dimensional measurement vector, $f_{k}(,, \boldsymbol{\alpha})$ and $\mathbf{h}_{k}\left(,, \boldsymbol{\lambda}_{k}\right)$ are known parametric vector functions depending on an unknown deterministic parameter vector ( $\boldsymbol{\alpha}$ and $\boldsymbol{\lambda}_{k}$ respectively). The process noise sequence $\left\{\mathbf{w}_{k}\right\}$ and the measurement noise sequence $\left\{\mathbf{v}_{k}\right\}$ are mutually independent white sequences described by known pdfs $p\left(\mathbf{w}_{k} \mid \boldsymbol{\beta}\right)$ and $p\left(\mathbf{v}_{k} \mid \boldsymbol{\mu}_{k}\right)$, respectively, depending on an unknown deterministic parameter vector ( $\boldsymbol{\beta}$ and $\boldsymbol{\mu}_{k}$ respectively). The noises are independent of the initial state $\mathbf{x}_{0}$ described by the known pdf $p\left(\mathbf{x}_{0} \mid \boldsymbol{\alpha}\right)$.

Let adopt the notational convention: $\forall l \leq k, \overline{\boldsymbol{\delta}}_{l: k}=$ $\left(\boldsymbol{\delta}_{l} ; \ldots ; \boldsymbol{\delta}_{k}\right)$ where all vectors are of same dimension. At time indexes $1,2, \ldots, k$ the dependency of the state and measurement on deterministic unknown parameters is as follows:

$$
\begin{aligned}
& \mid \begin{array}{ll}
\mathbf{x}_{1} \triangleq \mathbf{x}_{1}(\boldsymbol{\alpha}, \boldsymbol{\beta}) & =\mathbf{f}_{0}\left(\mathbf{x}_{0}, \mathbf{w}_{0}(\boldsymbol{\beta}), \boldsymbol{\alpha}\right) \\
\mathbf{y}_{1} \triangleq \mathbf{y}_{1}\left(\boldsymbol{\lambda}_{1}, \boldsymbol{\mu}_{1}, \boldsymbol{\alpha}, \boldsymbol{\beta}\right) & =\mathbf{h}_{1}\left(\mathbf{x}_{1}(\boldsymbol{\alpha}, \boldsymbol{\beta}), \mathbf{v}_{1}\left(\boldsymbol{\mu}_{1}\right), \boldsymbol{\lambda}_{1}\right)
\end{array} \\
& \mid \begin{array}{ll}
\mathbf{x}_{2} \triangleq \mathbf{x}_{2}(\boldsymbol{\alpha}, \boldsymbol{\beta}) & =\mathbf{f}_{1}\left(\mathbf{x}_{1}(\boldsymbol{\alpha}, \boldsymbol{\beta}), \mathbf{w}_{1}(\boldsymbol{\beta}), \boldsymbol{\alpha}\right) \\
\mathbf{y}_{2} \triangleq \mathbf{y}_{2}\left(\boldsymbol{\lambda}_{2}, \boldsymbol{\mu}_{2}, \boldsymbol{\alpha}, \boldsymbol{\beta}\right) & =\mathbf{h}_{2}\left(\mathbf{x}_{2}(\boldsymbol{\alpha}, \boldsymbol{\beta}), \mathbf{v}_{2}\left(\boldsymbol{\mu}_{2}\right), \boldsymbol{\lambda}_{2}\right)
\end{array}
\end{aligned}
$$

$\mid \begin{aligned} & \mathbf{x}_{k} \triangleq \mathbf{x}_{k}(\boldsymbol{\alpha}, \boldsymbol{\beta}) \quad=\mathbf{f}_{k-1}\left(\mathbf{x}_{k-1}(\boldsymbol{\alpha}, \boldsymbol{\beta}), \mathbf{w}_{k-1}(\boldsymbol{\beta}), \boldsymbol{\alpha}\right) \\ & \mathbf{y}_{k} \triangleq \mathbf{y}_{k}\left(\boldsymbol{\lambda}_{k}, \boldsymbol{\mu}_{k}, \boldsymbol{\alpha}, \boldsymbol{\beta}\right)=\mathbf{h}_{k}\left(\mathbf{x}_{k}(\boldsymbol{\alpha}, \boldsymbol{\beta}), \mathbf{v}_{k}\left(\boldsymbol{\mu}_{k}\right), \boldsymbol{\lambda}_{k}\right)\end{aligned}$

Therefore, the dependency of the state and measurement on deterministic unknown parameters on a horizon of $k$ points from the first measurement is as follows:

$$
k \geq 1: \mid \begin{array}{lc}
\overline{\mathbf{x}}_{0: k} \triangleq \overline{\mathbf{x}}_{0: k}(\boldsymbol{\alpha}, \boldsymbol{\beta}) \quad \triangleq \overline{\mathbf{x}}_{0: k}\left(\boldsymbol{\theta}_{0}\right) \\
\overline{\mathbf{y}}_{1: k} \triangleq \overline{\mathbf{y}}_{1: k}\left(\overline{\boldsymbol{\lambda}}_{1: k}, \overline{\boldsymbol{\mu}}_{1: k}, \boldsymbol{\alpha}, \boldsymbol{\beta}\right) \triangleq \overline{\mathbf{y}}_{1: k}\left(\boldsymbol{\theta}_{k}\right)
\end{array},
$$

$$
\boldsymbol{\theta}_{0}=(\boldsymbol{\alpha} ; \boldsymbol{\beta}), \quad k \geq 1: \boldsymbol{\theta}_{k}=\left(\overline{\boldsymbol{\lambda}}_{1: k} ; \overline{\boldsymbol{\mu}}_{1: k} ; \boldsymbol{\theta}_{0}\right),
$$

where $\boldsymbol{\theta}_{k}$ is the vector gathering all the unknown deterministic parameters. The state transition and the measurement pdfs depend on unknown deterministic parameters:

$p\left(\mathbf{x}_{k} \mid \mathbf{x}_{k-1}\right) \triangleq p\left(\mathbf{x}_{k} \mid \mathbf{x}_{k-1}, \boldsymbol{\theta}_{0}\right), p\left(\mathbf{y}_{k} \mid \mathbf{x}_{k}\right) \triangleq p\left(\mathbf{y}_{k} \mid \mathbf{x}_{k}, \boldsymbol{\lambda}_{k}, \boldsymbol{\mu}_{k}, \boldsymbol{\theta}_{0}\right)$,

and we suppose that both $p\left(\mathbf{x}_{k} \mid \mathbf{x}_{k-1}, \boldsymbol{\theta}_{0}\right)$ and $p\left(\mathbf{y}_{k} \mid \mathbf{x}_{k}, \boldsymbol{\lambda}_{k}, \boldsymbol{\mu}_{k}, \boldsymbol{\theta}_{0}\right)$ are twice differentiable with respect to all their arguments. Since (4) is a MDS:

$p\left(\overline{\mathbf{y}}_{1: k}, \overline{\mathbf{x}}_{0: k} \mid \boldsymbol{\theta}_{k}\right)=p\left(\mathbf{x}_{0} \mid \boldsymbol{\alpha}\right) \prod_{l=1}^{k} p\left(\mathbf{y}_{l} \mid \mathbf{x}_{l}, \boldsymbol{\lambda}_{l}, \boldsymbol{\mu}_{l}, \boldsymbol{\theta}_{0}\right) p\left(\mathbf{x}_{l} \mid \mathbf{x}_{l-1}, \boldsymbol{\theta}_{0}\right)$

From a theoretical point of view, we are primarily interested in the HFIM (2) on $(\mathbf{x} ; \boldsymbol{\theta}) \triangleq\left(\mathbf{x}_{k} ; \boldsymbol{\theta}_{k}\right)$ associated to the measurement vector $\mathbf{y} \triangleq \overline{\mathbf{y}}_{1: k}$ resulting from the set of $k$ measurements $\mathbf{y}_{1}, \ldots, \mathbf{y}_{k}$ :

$\mathbf{J}_{\left(\mathbf{x}_{k} ; \boldsymbol{\theta}_{k}\right)}=E_{\overline{\mathbf{y}}_{1: k}, \mathbf{x}_{k} \mid \boldsymbol{\theta}_{k}}\left[\frac{\partial \ln p\left(\overline{\mathbf{y}}_{1: k}, \mathbf{x}_{k} \mid \boldsymbol{\theta}_{k}\right)}{\partial\left(\mathbf{x}_{k} ; \boldsymbol{\theta}_{k}\right)} \frac{\partial \ln p\left(\overline{\mathbf{y}}_{1: k}, \mathbf{x}_{k} \mid \boldsymbol{\theta}_{k}\right)}{\partial\left(\mathbf{x}_{k} ; \boldsymbol{\theta}_{k}\right)^{T}}\right]$

which alternative formula is [3][4][5][6]:

$$
\mathbf{J}_{\left(\mathbf{x}_{k} ; \boldsymbol{\theta}_{k}\right)}=E_{\overline{\mathbf{y}}_{1: k}, \mathbf{x}_{k} \mid \boldsymbol{\theta}_{k}}\left[\frac{-\partial^{2} \ln p\left(\overline{\mathbf{y}}_{1: k}, \mathbf{x}_{k} \mid \boldsymbol{\theta}_{k}\right)}{\partial\left(\mathbf{x}_{k} ; \boldsymbol{\theta}_{k}\right) \partial\left(\mathbf{x}_{k} ; \boldsymbol{\theta}_{k}\right)^{T}}\right]
$$

Unfortunately the computation of (9) requires the derivation of the marginal pdf $p\left(\overline{\mathbf{y}}_{1: k}, \mathbf{x}_{k} \mid \boldsymbol{\theta}_{k}\right)$ from (8) which is generally mathematically intractable [2][5][12]. However an upper bound of (9) can be derived from the HFIM (2) on $\left(\mathbf{x} ; \boldsymbol{\theta}_{k}\right) \triangleq\left(\overline{\mathbf{x}}_{0: k} ; \boldsymbol{\theta}_{k}\right)$ associated to the measurement vector $\mathbf{y} \triangleq \overline{\mathbf{y}}_{1: k}[11]:$

$$
\mathbf{J}_{\left(\overline{\mathbf{x}}_{0: k} ; \boldsymbol{\theta}_{k}\right)}=E_{\overline{\mathbf{y}}_{1: k}, \overline{\mathbf{x}}_{0: k} \mid \boldsymbol{\theta}_{k}}\left[\frac{-\partial^{2} \ln p\left(\overline{\mathbf{y}}_{1: k}, \overline{\mathbf{x}}_{0: k} \mid \boldsymbol{\theta}_{k}\right)}{\partial\left(\overline{\mathbf{x}}_{0: k} ; \boldsymbol{\theta}_{k}\right) \partial\left(\overline{\mathbf{x}}_{0: k} ; \boldsymbol{\theta}_{k}\right)^{T}}\right] .
$$

Indeed, if we set $\mathbf{J}_{\left(\overline{\mathbf{x}}_{0: k} ; \boldsymbol{\theta}_{k}\right)}=\left[\begin{array}{ll}\mathbf{J}_{k}^{11} & \mathbf{B}_{k} \\ \mathbf{B}_{k}^{T} & \mathbf{C}_{k}\end{array}\right]$ where:

$$
\begin{aligned}
\mathbf{J}_{k}^{11} & =E_{\overline{\mathbf{y}}_{1: k}, \overline{\mathbf{x}}_{0: k} \mid \boldsymbol{\theta}_{k}}\left[-\frac{\partial^{2} \ln p\left(\overline{\mathbf{y}}_{1: k}, \overline{\mathbf{x}}_{0: k} \mid \boldsymbol{\theta}_{k}\right)}{\partial \overline{\mathbf{x}}_{0: k-1} \partial \overline{\mathbf{x}}_{0: k-1}^{T}}\right] \\
\mathbf{B}_{k} & =E_{\overline{\mathbf{y}}_{1: k}, \overline{\mathbf{x}}_{0: k} \mid \boldsymbol{\theta}_{k}}\left[-\frac{\partial^{2} \ln p\left(\overline{\mathbf{y}}_{1: k}, \overline{\mathbf{x}}_{0: k} \mid \boldsymbol{\theta}_{k}\right)}{\partial \overline{\mathbf{x}}_{0: k-1} \partial\left(\mathbf{x}_{k} ; \boldsymbol{\theta}_{k}\right)^{T}}\right], \\
\mathbf{C}_{k} & =E_{\overline{\mathbf{y}}_{1: k}, \overline{\mathbf{x}}_{0: k} \mid \boldsymbol{\theta}_{k}}\left[-\frac{\partial^{2} \ln p\left(\overline{\mathbf{y}}_{1: k}, \mathbf{x}_{k} \mid \boldsymbol{\theta}_{k}\right)}{\partial\left(\mathbf{x}_{k} ; \boldsymbol{\theta}_{k}\right) \partial\left(\mathbf{x}_{k} ; \boldsymbol{\theta}_{k}\right)^{T}}\right]
\end{aligned}
$$

then [11]:

$$
\begin{aligned}
\widetilde{\mathbf{J}}_{\left(\mathbf{x}_{k} ; \boldsymbol{\theta}_{k}\right)} \succeq \mathbf{J}_{\left(\mathbf{x}_{k} ; \boldsymbol{\theta}_{k}\right)} \Leftrightarrow \operatorname{HCRB}_{\left(\mathbf{x}_{k} ; \boldsymbol{\theta}_{k}\right)} \succeq \widetilde{\mathbf{J}}_{\left(\mathbf{x}_{k} ; \boldsymbol{\theta}_{k}\right)}^{-1}, \\
\widetilde{\mathbf{J}}_{\left(\mathbf{x}_{k} ; \boldsymbol{\theta}_{k}\right)}=\mathbf{C}_{k}-\mathbf{B}_{k}^{T}\left(\mathbf{J}_{k}^{11}\right)^{-1} \mathbf{B}_{k} .
\end{aligned}
$$

$\widetilde{\mathbf{J}}_{\left(\mathbf{x}_{k} ; \boldsymbol{\theta}_{k}\right)}^{-1}$ defines a looser (in comparison with $\operatorname{HCRB}_{\left(\mathbf{x}_{k} ; \boldsymbol{\theta}_{k}\right)}$ ) but general computable hybrid bound for discrete-time MDS pdf (8). However the computation of $\widetilde{\mathbf{J}}_{\left(\mathbf{x}_{k} ; \boldsymbol{\theta}_{k}\right)}^{-1}$ (11) may become rapidly computationally prohibitive and unstable since $\mathbf{J}_{k}^{11}$ and $\widetilde{\mathbf{J}}_{\left(\mathbf{x}_{k} ; \boldsymbol{\theta}_{k}\right)}$ are matrices with unbounded dimension as the time index $k$ increases (a $(P K \times P K)$ matrix and a $\left(\left(P+\operatorname{dim} \boldsymbol{\theta}_{k}\right) \times\left(P+\operatorname{dim} \boldsymbol{\theta}_{k}\right)\right)$ matrix, respectively $)$. Hence the need of a recursive form involving matrices with bounded dimensions. 


\section{A NEW RECURSIVE HCRB FOR MDS WITH}

TIME-VARIANT MEASUREMENT PARAMETERS

From a practical point of view, in estimation problem such (3)(4), at each time index $k$, the vector of parameters of interest is rather $\left(\mathbf{x}_{k} ; \boldsymbol{\omega}_{k}\right), \boldsymbol{\omega}_{k}=\left(\boldsymbol{\lambda}_{k} ; \boldsymbol{\mu}_{k} ; \boldsymbol{\alpha} ; \boldsymbol{\beta}\right)$, than $\left(\mathbf{x}_{k} ; \boldsymbol{\theta}_{k}\right)$. In that perspective, we can reparameterize $\left(\overline{\mathbf{x}}_{0: k} ; \boldsymbol{\theta}_{k}\right)$ as:

$$
\left(\begin{array}{c|c}
\overline{\mathbf{z}}_{0: k} \\
\boldsymbol{\theta}_{0}
\end{array}\right), \quad \begin{aligned}
& k=0: \mathbf{z}_{0}=\mathbf{x}_{0} \\
& k \geq 1: \mathbf{z}_{k}=\left(\mathbf{x}_{k} ; \boldsymbol{\lambda}_{k} ; \boldsymbol{\mu}_{k}\right)
\end{aligned},,
$$

and rearrange $\left(\mathbf{x}_{k} ; \boldsymbol{\theta}_{k}\right)$ as:

$$
\mathbf{v}_{k}=\left(\gamma_{k-1} ; \mathbf{z}_{k} ; \boldsymbol{\theta}_{0}\right), \quad \gamma_{k-1}=\left(\overline{\boldsymbol{\lambda}}_{1: k-1} ; \overline{\boldsymbol{\mu}}_{1: k-1}\right) .
$$

Then:

$$
\operatorname{HCRB}_{\mathbf{v}_{k}}=\left[\begin{array}{ll}
\operatorname{HCRB}_{\mathbf{v}_{k}}^{\gamma_{k-1}} & \operatorname{HCRB}_{\mathbf{v}_{k}}^{\gamma_{k-1},\left(\mathbf{z}_{k} ; \boldsymbol{\theta}_{0}\right)} \\
\operatorname{HCRB}_{\mathbf{v}_{k}}^{\left(\mathbf{z}_{k} ; \boldsymbol{\theta}_{0}\right), \boldsymbol{\gamma}_{k-1}} & \operatorname{HCRB}_{\mathbf{v}_{k}}^{\left(\mathbf{z}_{k} ; \boldsymbol{\theta}_{0}\right)}
\end{array}\right]
$$

and:

$$
\operatorname{HCRB}_{\left(\mathbf{x}_{k} ; \boldsymbol{\theta}_{k}\right)}^{\left(\mathbf{x}_{k} ; \boldsymbol{\omega}_{k}\right)}=\operatorname{HCRB}_{\mathbf{v}_{k}}^{\left(\mathbf{z}_{k} ; \boldsymbol{\theta}_{0}\right)} \succeq \widetilde{\mathbf{J}}_{\left(\mathbf{z}_{k} ; \boldsymbol{\theta}_{0}\right)}^{-1}
$$

where $\widetilde{\mathbf{J}}_{\left(\mathbf{z}_{k} ; \boldsymbol{\theta}_{0}\right)}$ can be decomposed into block matrices:

$$
\widetilde{\mathbf{J}}_{\left(\mathbf{z}_{k} ; \boldsymbol{\theta}_{0}\right)}=\left[\begin{array}{cc}
\widetilde{\mathbf{J}}_{k}^{\mathbf{z}_{k}, \mathbf{z}_{k}} & \widetilde{\mathbf{J}}_{k}^{\mathbf{z}_{k}, \boldsymbol{\theta}_{0}} \\
\widetilde{\mathbf{J}}_{k}^{\boldsymbol{\theta}_{0}, \mathbf{z}} & \widetilde{\mathbf{J}}_{k}^{\boldsymbol{\theta}_{0}, \boldsymbol{\theta}_{0}}
\end{array}\right]
$$

which obey the recursion:

$$
\begin{aligned}
& \widetilde{\mathbf{J}}_{k}^{\mathbf{z}_{k}, \mathbf{z}_{k}}= \mathbf{D}_{k-1}^{22}-\left(\mathbf{D}_{k-1}^{12}\right)^{T}\left(\mathbf{D}_{k-1}^{11}+\right. \\
&\left.\widetilde{\mathbf{J}}_{k-1}^{\mathbf{z}_{k-1}, \mathbf{z}_{k-1}}\right)^{-1} \mathbf{D}_{k-1}^{12} \\
& \widetilde{\mathbf{z}}_{k}, \boldsymbol{\theta}_{0}= \mathbf{D}_{k-1}^{23}-\left(\mathbf{D}_{k-1}^{12}\right)^{T}\left(\mathbf{D}_{k-1}^{11}+\widetilde{\mathbf{J}}_{k-1}^{\mathbf{z}_{k-1}, \mathbf{z}_{k-1}}\right)^{-1} \\
& \times\left(\mathbf{D}_{k-1}^{13}+\widetilde{\mathbf{J}}_{k-1}^{\mathbf{z}_{k-1}, \boldsymbol{\theta}_{0}}\right) \\
& \widetilde{\mathbf{J}}_{k}^{\boldsymbol{\theta}_{0}, \boldsymbol{\theta}_{0}}= \mathbf{D}_{k-1}^{33}+\widetilde{\mathbf{J}}_{k-1}^{\boldsymbol{\theta}_{0}, \boldsymbol{\theta}_{0}}-\left(\mathbf{D}_{k-1}^{13}+\widetilde{\mathbf{J}}_{k-1}^{\mathbf{z}_{k-1}, \boldsymbol{\theta}_{0}}\right)^{T} \\
& \times\left(\mathbf{D}_{k-1}^{11}+\widetilde{\mathbf{J}}_{k-1}^{\mathbf{z}_{k-1}, \mathbf{z}_{k-1}}\right)^{-1}\left(\mathbf{D}_{k-1}^{13}+\widetilde{\mathbf{J}}_{k-1}^{\mathbf{z}_{k-1}, \boldsymbol{\theta}_{0}}\right)
\end{aligned}
$$

where:

$$
\begin{aligned}
& \mathbf{D}_{k-1}^{11}=E_{\mathbf{x}_{k}, \mathbf{x}_{k-1} \mid \boldsymbol{\theta}_{0}}\left[-\frac{\partial^{2} \ln p\left(\mathbf{x}_{k} \mid \mathbf{x}_{k-1}, \boldsymbol{\theta}_{0}\right)}{\partial \mathbf{z}_{k-1} \partial \mathbf{z}_{k-1}^{T}}\right] \\
& \mathbf{D}_{k-1}^{12}=E_{\mathbf{x}_{k}, \mathbf{x}_{k-1} \mid \boldsymbol{\theta}_{0}}\left[-\frac{\partial^{2} \ln p\left(\mathbf{x}_{k} \mid \mathbf{x}_{k-1}, \boldsymbol{\theta}_{0}\right)}{\partial \mathbf{z}_{k-1} \partial \mathbf{z}_{k}^{T}}\right] \\
& \mathbf{D}_{k-1}^{22}=E_{\mathbf{y}_{k}, \mathbf{x}_{k} \mid \boldsymbol{\lambda}_{k}, \boldsymbol{\mu}_{k}, \boldsymbol{\theta}_{0}}\left[-\frac{\partial^{2} \ln p\left(\mathbf{y}_{k} \mid \mathbf{x}_{k}, \boldsymbol{\lambda}_{k}, \boldsymbol{\mu}_{k}, \boldsymbol{\theta}_{0}\right)}{\partial \mathbf{z}_{k} \partial \mathbf{z}_{k}^{T}}\right] \\
& +E_{\mathbf{x}_{k}, \mathbf{x}_{k-1} \mid \boldsymbol{\theta}_{0}}\left[-\frac{\partial^{2} \ln p\left(\mathbf{x}_{k} \mid \mathbf{x}_{k-1}, \boldsymbol{\theta}_{0}\right)}{\partial \mathbf{z}_{k} \partial \mathbf{z}_{k}^{T}}\right] \\
& \mathbf{D}_{k-1}^{13}=E_{\mathbf{x}_{k}, \mathbf{x}_{k-1} \mid \boldsymbol{\theta}_{0}}\left[-\frac{\partial^{2} \ln p\left(\mathbf{x}_{k} \mid \mathbf{x}_{k-1}, \boldsymbol{\theta}_{0}\right)}{\partial \mathbf{z}_{k-1} \partial \boldsymbol{\theta}_{0}^{T}}\right] \\
& \mathbf{D}_{k-1}^{23}=E_{\mathbf{y}_{k}, \mathbf{x}_{k} \mid \boldsymbol{\lambda}_{k}, \boldsymbol{\mu}_{k}, \boldsymbol{\theta}_{0}}\left[-\frac{\partial^{2} \ln p\left(\mathbf{y}_{k} \mid \mathbf{x}_{k}, \boldsymbol{\lambda}_{k}, \boldsymbol{\mu}_{k}, \boldsymbol{\theta}_{0}\right)}{\partial \mathbf{z}_{k} \partial \boldsymbol{\theta}_{0}^{T}}\right] \\
& +E_{\mathbf{x}_{k}, \mathbf{x}_{k-1} \mid \boldsymbol{\theta}_{0}}\left[-\frac{\partial^{2} \ln p\left(\mathbf{x}_{k} \mid \mathbf{x}_{k-1}, \boldsymbol{\theta}_{0}\right)}{\partial \mathbf{z}_{k} \partial \boldsymbol{\theta}_{0}^{T}}\right] \\
& \mathbf{D}_{k-1}^{33}=E_{\mathbf{y}_{k}, \mathbf{x}_{k} \mid \boldsymbol{\lambda}_{k}, \boldsymbol{\mu}_{k}, \boldsymbol{\theta}_{0}}\left[-\frac{\partial^{2} \ln p\left(\mathbf{y}_{k} \mid \mathbf{x}_{k}, \boldsymbol{\lambda}_{k}, \boldsymbol{\mu}_{k}, \boldsymbol{\theta}_{0}\right)}{\partial \boldsymbol{\theta}_{0} \partial \boldsymbol{\theta}_{0}^{T}}\right] \\
& +E_{\mathbf{x}_{k}, \mathbf{x}_{k-1} \mid \boldsymbol{\theta}_{0}}\left[-\frac{\partial^{2} \ln p\left(\mathbf{x}_{k} \mid \mathbf{x}_{k-1}, \boldsymbol{\theta}_{0}\right)}{\partial \boldsymbol{\theta}_{0} \partial \boldsymbol{\theta}_{0}^{T}}\right] \\
& \widetilde{\mathbf{J}}_{\left(\mathbf{z}_{0} ; \boldsymbol{\theta}_{0}\right)}=\mathbf{J}_{\left(\mathbf{z}_{0} ; \boldsymbol{\theta}_{0}\right)}=E_{\mathbf{x}_{0} \mid \boldsymbol{\theta}_{0}}\left[\frac{-\partial^{2} \ln p\left(\mathbf{x}_{0} \mid \boldsymbol{\alpha}\right)}{\partial\left(\mathbf{x}_{0} ; \boldsymbol{\theta}_{0}\right) \partial\left(\mathbf{x}_{0} ; \boldsymbol{\theta}_{0}\right)^{T}}\right]
\end{aligned}
$$

Proof:

The first step consists in reformulating (11)(12) after the rearrangement (14) leading to:

$$
\begin{gathered}
\widetilde{\mathbf{J}}_{\mathbf{v}_{k}} \succeq \mathbf{J}_{\mathbf{v}_{k}}, \quad \widetilde{\mathbf{J}}_{\mathbf{v}_{k}}=\underline{\mathbf{C}}_{k}-\underline{\mathbf{B}}_{k}^{T}\left(\mathbf{J}_{k}^{11}\right)^{-1} \underline{\mathbf{B}}_{k}, \\
\mathbf{J}_{k}^{11}=E_{\overline{\mathbf{y}}_{1: k}, \overline{\mathbf{x}}_{0: k} \mid \boldsymbol{\theta}_{k}}\left[-\frac{\partial^{2} \ln p\left(\overline{\mathbf{y}}_{1: k}, \overline{\mathbf{x}}_{0: k} \mid \boldsymbol{\theta}_{k}\right)}{\partial \overline{\mathbf{x}}_{0: k-1} \partial \overline{\mathbf{x}}_{0: k-1}^{T}}\right] \\
\underline{\mathbf{B}}_{k}=E_{\overline{\mathbf{y}}_{1: k}, \overline{\mathbf{x}}_{0: k} \mid \boldsymbol{\theta}_{k}}\left[-\frac{\partial^{2} \ln p\left(\overline{\mathbf{y}}_{1: k}, \overline{\mathbf{x}}_{0: k} \mid \boldsymbol{\theta}_{k}\right)}{\partial \overline{\mathbf{x}}_{0: k-1} \partial \mathbf{v}_{k}^{T}}\right] \\
\underline{\mathbf{C}}_{k}=E_{\overline{\mathbf{y}}_{1: k}, \overline{\mathbf{x}}_{0: k} \mid \boldsymbol{\theta}_{k}}\left[-\frac{\partial^{2} \ln p\left(\overline{\mathbf{y}}_{1: k}, \mathbf{x}_{k} \mid \boldsymbol{\theta}_{k}\right)}{\partial \mathbf{v}_{k} \partial \mathbf{v}_{k}^{T}}\right]
\end{gathered}
$$

Let $\mathbf{u}_{k}=\left(\overline{\mathbf{x}}_{0: k-1} ; \boldsymbol{\gamma}_{k-1} ; \mathbf{z}_{k} ; \boldsymbol{\theta}_{0}\right) \triangleq\left(\overline{\mathbf{x}}_{0: k-1} ; \mathbf{v}_{k}\right)$, that is $\left(\overline{\mathbf{x}}_{0: k} ; \boldsymbol{\theta}_{k}\right)$ after rearrangement, then:

$\mathbf{J}_{\mathbf{u}_{k}}=E_{\overline{\mathbf{y}}_{1: k}, \overline{\mathbf{x}}_{0: k} \mid \boldsymbol{\theta}_{k}}\left[-\frac{\partial^{2} \ln p\left(\overline{\mathbf{y}}_{1: k}, \overline{\mathbf{x}}_{0: k} \mid \boldsymbol{\theta}_{k}\right)}{\partial \mathbf{u}_{k} \partial \mathbf{u}_{k}^{T}}\right]=\left[\begin{array}{cc}\mathbf{J}_{k}^{11} & \underline{\mathbf{B}}_{k} \\ \underline{\mathbf{B}}_{k}^{T} & \underline{\mathbf{C}}_{k}\end{array}\right]$.

Therefore, using block matrix inversion:

$$
\begin{aligned}
\mathbf{H C R B}_{\mathbf{u}_{k}}=\mathbf{J}_{\mathbf{u}_{k}}^{-1} & =\left[\begin{array}{ll}
\mathbf{H C R B}_{\mathbf{u}_{k}, 1}^{\overline{\mathbf{x}}_{0:-1}} & \mathbf{H C R B}_{\mathbf{u}_{k}}^{\overline{\mathbf{x}}_{0: k-1}, \mathbf{v}_{k}} \\
\mathbf{H C R B}_{\mathbf{u}_{k}}^{\mathbf{v}_{0: k-1}} & \mathbf{H C R B}_{\mathbf{u}_{k}}^{\mathbf{v}_{k}}
\end{array}\right], \\
\mathbf{H C R B}_{\mathbf{u}_{k}}^{\mathbf{v}_{k}} & =\left(\underline{\mathbf{C}}_{k}-\underline{\mathbf{B}}_{k}^{T}\left(\mathbf{J}_{k}^{11}\right)^{-1} \underline{\mathbf{B}}_{k}\right)^{-1}=\widetilde{\mathbf{J}}_{\mathbf{v}_{k}}^{-1} .
\end{aligned}
$$

Additionally, according to (13)(14):

$\overline{\mathbf{z}}_{0: k-1}=\mathbf{P}_{0: k-1}\left(\begin{array}{c}\overline{\mathbf{x}}_{0: k-1} \\ \boldsymbol{\gamma}_{k-1}\end{array}\right) \Rightarrow\left(\begin{array}{c}\overline{\mathbf{z}}_{0: k-1} \\ \left(\begin{array}{c}\mathbf{z}_{k} \\ \boldsymbol{\theta}_{0}\end{array}\right)\end{array}\right)=\left[\begin{array}{cc}\mathbf{P}_{0: k-1} & \mathbf{0} \\ \mathbf{0} & \mathbf{I}\end{array}\right]$,

where $\mathbf{P}_{0: k}$ is a permutation matrix; therefore:

$\mathbf{H C R B}_{\mathbf{u}_{k}}=\left[\begin{array}{cc}\mathbf{P}_{0: k-1}^{T} & \mathbf{0} \\ \mathbf{0} & \mathbf{I}\end{array}\right] \mathbf{H C R B}_{\left(\overline{\mathbf{z}}_{0: k} ; \boldsymbol{\theta}_{0}\right)}\left[\begin{array}{cc}\mathbf{P}_{0: k-1} & \mathbf{0} \\ \mathbf{0} & \mathbf{I}\end{array}\right]$,

leading to the key identity:

$$
\operatorname{HCRB}_{\mathbf{u}_{k}}^{\left(\mathbf{z}_{k} ; \boldsymbol{\theta}_{0}\right)}=\operatorname{HCRB}_{\left(\mathbf{z}_{0: k} ; \boldsymbol{\theta}_{0}\right)}^{\left(\mathbf{z}_{k} ; \boldsymbol{\theta}_{0}\right)}
$$

Indeed, (21) yields:

$$
\mathbf{J}_{\mathbf{v}_{k}}^{-1} \succeq \widetilde{\mathbf{J}}_{\mathbf{v}_{k}}^{-1} \Leftrightarrow \mathbf{H C R B}_{\mathbf{v}_{k}} \succeq \mathbf{H C R B}_{\mathbf{u}_{k}}^{\mathbf{v}_{k}},
$$

that is, in particular:

$$
\operatorname{HCRB}_{\mathbf{v}_{k}}^{\left(\mathbf{z}_{k} ; \boldsymbol{\theta}_{0}\right)} \succeq \operatorname{HCRB}_{\mathbf{u}_{k}}^{\left(\mathbf{z}_{k} ; \boldsymbol{\theta}_{0}\right)}=\operatorname{HCRB}_{\left(\overline{\mathbf{z}}_{0: k} ; \boldsymbol{\theta}_{0}\right)}^{\left(\mathbf{z}_{\mathbf{A}} ; \boldsymbol{\theta}_{0}\right)} .
$$

The second step consists in showing that

$$
\widetilde{\mathbf{J}}_{\left(\mathbf{z}_{k} ; \boldsymbol{\theta}_{0}\right)}=\left(\operatorname{HCRB}_{\left(\overline{\mathbf{z}}_{0: k} ; \boldsymbol{\theta}_{0}\right)}^{\left(\mathbf{z}_{k} ; \boldsymbol{\theta}_{0}\right)}\right)^{-1}=\left[\begin{array}{cc}
\widetilde{\mathbf{J}}_{k}^{\mathbf{z}_{k}, \mathbf{z}_{k}} & \widetilde{\mathbf{J}}_{k}^{\mathbf{z}_{k}, \boldsymbol{\theta}_{0}} \\
\widetilde{\mathbf{J}}_{k}^{\boldsymbol{\theta}_{0}, \mathbf{z}_{k}} & \widetilde{\mathbf{J}}_{k}^{\boldsymbol{\theta}_{0}, \boldsymbol{\theta}_{0}}
\end{array}\right]
$$

obey the recursion defined by (17-20). First $\mathbf{J}_{\left(\overline{\mathbf{z}}_{0: k} ; \boldsymbol{\theta}_{0}\right)}$ can be broken down as:

$$
\begin{aligned}
& \mathbf{J}_{\left(\overline{\mathbf{z}}_{0: k} ; \boldsymbol{\theta}_{0}\right)}=\left[\begin{array}{ccc}
\mathbf{J}_{k}^{11} & \mathbf{J}_{k}^{12} & \mathbf{J}_{k}^{13} \\
\left(\mathbf{J}_{k}^{12}\right)^{T} & \mathbf{J}_{k}^{22} & \mathbf{J}_{k}^{23} \\
\left(\mathbf{J}_{k}^{13}\right)^{T} & \left(\mathbf{J}_{k}^{23}\right)^{T} & \mathbf{J}_{k}^{33}
\end{array}\right], \\
& \mathbf{J}_{k}^{11}=E_{\overline{\mathbf{y}}_{1: k}, \overline{\mathbf{x}}_{0: k} \mid \boldsymbol{\theta}_{k}}\left[-\frac{\partial^{2} \ln p\left(\overline{\mathbf{y}}_{1: k}, \overline{\mathbf{x}}_{0: k} \mid \boldsymbol{\theta}_{k}\right)}{\partial \overline{\mathbf{z}}_{0: k-1} \partial \overline{\mathbf{z}}_{0: k-1}^{T}}\right], \\
& \mathbf{J}_{k}^{12}=E_{\overline{\mathbf{y}}_{1: k}, \overline{\mathbf{x}}_{0: k} \mid \boldsymbol{\theta}_{k}}\left[-\frac{\partial^{2} \ln p\left(\overline{\mathbf{y}}_{1: k}, \mathbf{\mathbf { x }}_{0: k} \mid \boldsymbol{\theta}_{k}\right)}{\partial \overline{\mathbf{z}}_{0: k-1} \partial \mathbf{z}_{k}^{T}}\right], \\
& \mathbf{J}_{k}^{13}=E_{\overline{\mathbf{y}}_{1: k}, \overline{\mathbf{x}}_{0: k} \mid \boldsymbol{\theta}_{k}}\left[-\frac{\partial^{2} \ln p\left(\overline{\mathbf{y}}_{1: k}, \overline{\mathbf{x}}_{k: k} \mid \boldsymbol{\theta}_{k}\right)}{\partial \overline{\mathbf{z}}_{0: k-1} \partial \boldsymbol{\theta}_{0}^{T}}\right], \\
& \mathbf{J}_{k}^{22}=E_{\overline{\mathbf{y}}_{1: k}, \overline{\mathbf{x}}_{0: k} \mid \boldsymbol{\theta}_{k}}-\frac{\partial^{2} \ln p\left(\overline{\mathbf{y}}_{1: k}, \overline{\mathbf{x}}_{0: k} \mid \boldsymbol{\theta}_{k}\right)}{\partial \mathbf{z}_{k} \partial \mathbf{z}_{k}^{T}}, \text {, } \\
& \left.\mathbf{J}_{k}^{23}=E_{\overline{\mathbf{y}}_{1: k}, \overline{\mathbf{x}}_{0: k} \mid \boldsymbol{\theta}_{k}}-\frac{\partial^{2} \ln p\left(\overline{\mathbf{y}}_{1: k}, \overline{\mathbf{x}}_{0: k} \mid \boldsymbol{\theta}_{k}\right)}{\partial \mathbf{z}_{k} \partial \boldsymbol{\theta}_{0}^{T}}\right], \\
& \mathbf{J}_{k}^{33}=E_{\overline{\mathbf{y}}_{1: k}, \overline{\mathbf{x}}_{0: k} \mid \boldsymbol{\theta}_{k}}\left[-\frac{\partial^{2} \ln p\left(\overline{\mathbf{y}}_{1: k}, \overline{\mathbf{x}}_{0: k} \mid \boldsymbol{\theta}_{k}\right)}{\partial \boldsymbol{\theta}_{0}^{T} \partial \boldsymbol{\theta}_{0}^{T}}\right] .
\end{aligned}
$$


Therefore, using block matrix inversion:

$$
\widetilde{\mathbf{J}}_{\left(\mathbf{z}_{k} ; \boldsymbol{\theta}_{0}\right)}=\left[\begin{array}{cc}
\mathbf{J}_{k}^{22} & \mathbf{J}_{k}^{23} \\
\mathbf{J}_{k}^{32} & \mathbf{J}_{k}^{33}
\end{array}\right]-\left[\begin{array}{c}
\mathbf{J}_{k}^{21} \\
\mathbf{J}_{k}^{31}
\end{array}\right]\left[\mathbf{J}_{k}^{11}\right]^{-1}\left[\begin{array}{ll}
\mathbf{J}_{k}^{12} & \mathbf{J}_{k}^{13}
\end{array}\right]
$$

that is:

$$
\mid \begin{aligned}
& \widetilde{\mathbf{J}}_{k}^{\mathbf{z}_{k}, \mathbf{z}_{k}}=\mathbf{J}_{k}^{22}-\mathbf{J}_{k}^{21}\left(\mathbf{J}_{k-1}^{11}\right)^{-1} \mathbf{J}_{k}^{12}, \\
& \widetilde{\mathbf{J}}_{k}^{\boldsymbol{\theta}_{0}, \mathbf{z}}=\mathbf{J}_{k}^{23}-\mathbf{J}_{k}^{21}\left(\mathbf{J}_{k}^{11}\right)^{-1} \mathbf{J}_{k}^{13}, \\
& \widetilde{\mathbf{J}}_{k}^{\boldsymbol{\theta}_{0}, \boldsymbol{\theta}_{0}}=\mathbf{J}_{k}^{33}-\mathbf{J}_{k}^{31}\left(\mathbf{J}_{k}^{11}\right)^{-1} \mathbf{J}_{k}^{13} .
\end{aligned}
$$

Moreover, for MDS, (8) leads to:

$$
\begin{array}{r}
p\left(\overline{\mathbf{y}}_{1: k}, \overline{\mathbf{x}}_{0: k} \mid \boldsymbol{\theta}_{k}\right)=p\left(\mathbf{y}_{k} \mid \mathbf{x}_{k}, \boldsymbol{\lambda}_{k}, \boldsymbol{\mu}_{k}, \boldsymbol{\theta}_{0}\right) p\left(\mathbf{x}_{k} \mid \mathbf{x}_{k-1}, \boldsymbol{\theta}_{0}\right) \\
\times p\left(\overline{\mathbf{y}}_{1: k-1}, \overline{\mathbf{x}}_{0: k-1} \mid \boldsymbol{\theta}_{k-1}\right)
\end{array}
$$

yielding:

$$
\begin{aligned}
& \mathbf{J}_{k}^{11}=\left[\begin{array}{cc}
\mathbf{J}_{k-1}^{11} & \mathbf{J}_{k-1}^{12} \\
\mathbf{J}_{k-1}^{21} & \mathbf{J}_{k-1}^{22}+\mathbf{D}_{k-1}^{11}
\end{array}\right], \quad \mathbf{J}_{k}^{12}=\left[\begin{array}{c}
\mathbf{0} \\
\mathbf{D}_{k-1}^{12}
\end{array}\right], \\
& \mathbf{J}_{k}^{22}=\mathbf{D}_{k-1}^{22}, \quad \mathbf{J}_{k}^{13}=\left[\begin{array}{c}
\mathbf{J}_{k-1}^{13} \\
\mathbf{J}_{k-1}^{23}+\mathbf{D}_{k-1}^{13}
\end{array}\right], \\
& \mathbf{J}_{k}^{23}=\mathbf{D}_{k-1}^{23}, \quad \mathbf{J}_{k}^{33}=\mathbf{J}_{k-1}^{33}+\mathbf{D}_{k-1}^{33},
\end{aligned}
$$

where $\mathbf{D}_{k-1}^{i j}$ are given by (18-19). Last, using once again block matrix inversion:

$$
\begin{aligned}
& \left(\mathbf{J}_{k}^{11}\right)^{-1}=\left[\begin{array}{cc}
\boldsymbol{\Phi}_{k-1} & -\boldsymbol{\Gamma}_{k-1} \boldsymbol{\Delta}_{k-1}^{-1} \\
-\boldsymbol{\Delta}_{k-1}^{-1} \boldsymbol{\Gamma}_{k-1}^{T} & \boldsymbol{\Delta}_{k-1}^{-1}
\end{array}\right], \\
& \boldsymbol{\Delta}_{k-1}=\mathbf{D}_{k-1}^{11}+\mathbf{J}_{k-1}^{22}-\mathbf{J}_{k-1}^{21}\left(\mathbf{J}_{k-1}^{11}\right)^{-1} \mathbf{J}_{k-1}^{12}, \\
& \boldsymbol{\Gamma}_{k-1}=\left(\mathbf{J}_{k-1}^{11}\right)^{-1} \mathbf{J}_{k-1}^{12}, \\
& \boldsymbol{\Phi}_{k-1}=\left(\mathbf{J}_{k-1}^{11}\right)^{-1}+\boldsymbol{\Gamma}_{k-1} \boldsymbol{\Delta}_{k-1}^{-1} \boldsymbol{\Gamma}_{k-1}^{T} .
\end{aligned}
$$

Then noting that $\boldsymbol{\Delta}_{k-1}=\mathbf{D}_{k-1}^{11}+\widetilde{\mathbf{J}}_{k-1}^{\mathbf{z}_{k-1}, \mathbf{z}_{k-1}}$, a few additional lines of calculus allows to show that equivalent forms of $\widetilde{\mathbf{J}}_{k}^{\mathbf{z}_{k}, \mathbf{z}_{k}}, \widetilde{\mathbf{J}}_{k}^{\boldsymbol{\theta}_{0}, \boldsymbol{\theta}_{0}}$ and $\widetilde{\mathbf{J}}_{k}^{\boldsymbol{\theta}_{0}, \mathbf{z}}$ in (24) are given by (17).

\section{FURTHER CONSIDERATIONS}

First, some special cases of interest can be easily derived by updating the definitions of $\boldsymbol{\theta}_{k}, p\left(\mathbf{x}_{k} \mid \mathbf{x}_{k-1}\right), p\left(\mathbf{y}_{k} \mid \mathbf{x}_{k}\right)$ and $p\left(\mathbf{x}_{0} \mid \boldsymbol{\alpha}\right)$ accordingly.

A first case of interest is $\boldsymbol{\theta}_{k}=\boldsymbol{\theta}_{0}$, i.e. the measurements do not depend on deterministic parameters, then $\mathbf{z}_{k}=\mathbf{x}_{k}$, $p\left(\mathbf{x}_{k} \mid \mathbf{x}_{k-1}\right) \triangleq p\left(\mathbf{x}_{k} \mid \mathbf{x}_{k-1}, \boldsymbol{\theta}_{0}\right), p\left(\mathbf{y}_{k} \mid \mathbf{x}_{k}\right) \triangleq p\left(\mathbf{y}_{k} \mid \mathbf{x}_{k}, \boldsymbol{\theta}_{0}\right)$, and (15-16) reduce to:

$\operatorname{HCRB}_{\left(\mathbf{x}_{k} ; \boldsymbol{\theta}_{0}\right)} \succeq \widetilde{\mathbf{J}}_{\left(\mathbf{x}_{k} ; \boldsymbol{\theta}_{0}\right)}^{-1}, \widetilde{\mathbf{J}}_{\left(\mathbf{x}_{k} ; \boldsymbol{\theta}_{0}\right)}=\left[\begin{array}{cc}\widetilde{\mathbf{J}}_{k}^{\mathbf{x}_{k}, \mathbf{x}_{k}} & \widetilde{\mathbf{J}}_{k}^{\mathbf{x}_{k}, \boldsymbol{\theta}_{0}} \\ \widetilde{\mathbf{J}}_{k}^{\boldsymbol{\theta}_{0}, \mathbf{x}_{k}} & \widetilde{\mathbf{J}}_{k}^{\boldsymbol{\theta}_{0}, \boldsymbol{\theta}_{0}}\end{array}\right]$,

which are $[11,(7)(10)]$ where $\boldsymbol{\theta} \triangleq(\boldsymbol{\alpha} ; \boldsymbol{\beta})$, and (17-20) reduce to $[11,(11-14)]$.

A second case of interest is $\boldsymbol{\theta}_{k}=\varnothing$, i.e. there is no unknown deterministic parameter, then $\mathbf{z}_{k}=\mathbf{x}_{k}, p\left(\mathbf{x}_{k} \mid \mathbf{x}_{k-1}\right) \triangleq$ $p\left(\mathbf{x}_{k} \mid \mathbf{x}_{k-1}\right), p\left(\mathbf{y}_{k} \mid \mathbf{x}_{k}\right) \triangleq p\left(\mathbf{y}_{k} \mid \mathbf{x}\right)$, and (15-16) and (17-20) reduce to:

$$
\begin{gathered}
\mathbf{H C R B}_{\mathbf{x}_{k}} \succeq \widetilde{\mathbf{J}}_{\mathbf{x}_{k}}^{-1}, \quad \widetilde{\mathbf{J}}_{\mathbf{x}_{k}}=\widetilde{\mathbf{J}}_{k}^{\mathbf{x}_{k}, \mathbf{x}_{k}}, \\
\widetilde{\mathbf{J}}_{k}^{\mathbf{x}_{k}, \mathbf{x}_{k}}=\mathbf{D}_{k-1}^{22}-\left(\mathbf{D}_{k-1}^{12}\right)^{T}\left(\mathbf{D}_{k-1}^{11}+\widetilde{\mathbf{J}}_{k-1}^{\mathbf{x}_{k-1}, \mathbf{x}_{k-1}}\right)^{-1} \mathbf{D}_{k-1}^{12},
\end{gathered}
$$

$$
\begin{aligned}
& \mathbf{D}_{k-1}^{11}=E_{\mathbf{x}_{k}, \mathbf{x}_{k-1}}\left[\frac{-\partial^{2} \ln p\left(\mathbf{x}_{k} \mid \mathbf{x}_{k-1}\right)}{\partial \mathbf{x}_{k-1} \partial \mathbf{x}_{k-1}^{T}}\right], \\
& \mathbf{D}_{k-1}^{12}=E_{\mathbf{x}_{k}, \mathbf{x}_{k-1}}\left[\frac{-\partial^{2} \ln p\left(\mathbf{x}_{k} \mid \mathbf{x}_{k-1}\right)}{\partial \mathbf{x}_{k-1} \partial \mathbf{x}_{k}^{T}}\right], \\
& \mathbf{D}_{k-1}^{22}=E_{\mathbf{y}_{k}, \mathbf{x}_{k}}\left[\frac{-\partial^{2} \ln p\left(\mathbf{y}_{k} \mid \mathbf{x}_{k}\right)}{\partial \mathbf{x}_{k} \partial \mathbf{x}_{k}^{T}}\right]+E_{\mathbf{x}_{k}, \mathbf{x}_{k-1}}\left[\frac{-\partial^{2} \ln p\left(\mathbf{x}_{k} \mid \mathbf{x}_{k-1}\right)}{\partial \mathbf{x}_{k} \partial \mathbf{x}_{k}^{T}}\right]
\end{aligned}
$$

and $\widetilde{\mathbf{J}}_{\left(\mathbf{z}_{0} ; \boldsymbol{\theta}_{0}\right)} \triangleq \mathbf{J}_{\mathbf{x}_{0}}$, which are (21-25) in [12].

A third case of interest is $\boldsymbol{\theta}_{0}=\varnothing$, i.e. the state does not depend on deterministic parameters, then $p\left(\mathbf{x}_{k} \mid \mathbf{x}_{k-1}\right) \triangleq$ $p\left(\mathbf{x}_{k} \mid \mathbf{x}_{k-1}\right), p\left(\mathbf{y}_{k} \mid \mathbf{x}_{k}\right) \triangleq p\left(\mathbf{y}_{k} \mid \mathbf{z}_{k}\right)$, and (15-16) and (17-20) reduce to:

$$
\begin{gathered}
\mathbf{H C R B}_{\mathbf{z}_{k}} \succeq \widetilde{\mathbf{J}}_{\mathbf{z}_{k}}^{-1}, \quad \widetilde{\mathbf{J}}_{\mathbf{z}_{k}}=\widetilde{\mathbf{J}}_{k}^{\mathbf{z}_{k}, \mathbf{z}_{k}}, \\
\widetilde{\mathbf{J}}_{k}^{\mathbf{z}_{k}, \mathbf{z}_{k}}=\mathbf{D}_{k-1}^{22}-\left(\mathbf{D}_{k-1}^{12}\right)^{T}\left(\mathbf{D}_{k-1}^{11}+\widetilde{\mathbf{J}}_{k-1}^{\mathbf{z}_{k-1}, \mathbf{z}_{k-1}}\right)^{-1} \mathbf{D}_{k-1}^{12}, \\
\mathbf{D}_{k-1}^{11}=E_{\mathbf{x}_{k}, \mathbf{x}_{k-1}}\left[\frac{-\partial^{2} \ln p\left(\mathbf{x}_{k} \mid \mathbf{x}_{k-1}\right)}{\partial \mathbf{z}_{k-1} \partial \mathbf{z}_{k-1}^{T}}\right], \\
\mathbf{D}_{k-1}^{12}=E_{\mathbf{x}_{k}, \mathbf{x}_{k-1}}\left[\frac{-\partial^{2} \ln p\left(\mathbf{x}_{k} \mid \mathbf{x}_{k-1}\right)}{\partial \mathbf{z}_{k-1} \partial \mathbf{z}_{k}^{T}}\right], \\
\mathbf{D}_{k-1}^{22}=E_{\mathbf{y}_{k}, \mathbf{x}_{k} \mid \boldsymbol{\lambda}_{k}, \boldsymbol{\mu}_{k}\left[\frac{-\partial^{2} \ln p\left(\mathbf{y}_{k} \mid \mathbf{z}_{k}\right)}{\partial \mathbf{z}_{k} \partial \mathbf{z}_{k}^{T}}\right]}+E_{\mathbf{y}_{k}, \mathbf{x}_{k} \mid \boldsymbol{\lambda}_{k}, \boldsymbol{\mu}_{k}}\left[\frac{-\partial^{2} \ln p\left(\mathbf{x}_{k} \mid \mathbf{x}_{k-1}\right)}{\partial \mathbf{z}_{k} \partial \mathbf{z}_{k}^{T}}\right]
\end{gathered}
$$

and $\widetilde{\mathbf{J}}_{\mathbf{z}_{0}} \triangleq \mathbf{J}_{\mathbf{x}_{0}}$ which is a generalization of [12].

Second, it is worth noting that, at each time index $k$, there is no particular requirements on the dimension of $\boldsymbol{\lambda}_{k}$ or $\boldsymbol{\mu}_{k}$; in particular it is possible to have $\boldsymbol{\lambda}_{l}=\varnothing$ and/or $\boldsymbol{\mu}_{l}=\varnothing$ for a subset of time index $l \in\left\{l_{1}, l_{2}, \ldots, l_{J} \leq k\right\}$, what means that the new HCRB (15) allows to take into account a mixture of measurements where the deterministic parameters are either known or unknown (which is not possible with the HCRB derived in [11]).

\section{REFERENCES}

[1] D. Simon, Optimal State Estimation: Kalman, H-infinity, and Nonlinear Approaches, Wiley InterScience, 2006

[2] H. L. Van Trees and K. L. Bell, Eds., Bayesian Bounds for Parameter Estimation and Nonlinear Filtering/Tracking. Wiley/IEEE Press, 2007.

[3] Y. Rockah and P. Schultheiss, "Array shape calibration using sources in unknown locations-part I: Far-field sources," IEEE Trans. on ASSP, 35(3):286-299, 1987.

[4] S. Bay, B. Geller, A. Renaux, J.-P. Barbot, and J.-M. Brossier, "On the hybrid Cramér-Rao bound and its application to dynamical phase estimation," IEEE SP Letters, 15:453-456, 2008

[5] Y. Noam and H. Messer, "Notes on the tightness of the hybrid CramérRao lower bound," IEEE Trans. on SP, 57(6):2074-2084, 2009.

[6] I. Reuven and H. Messer, "A Barankin-type lower bound on the estimation error of a hybrid parameter vector," IEEE Trans on IT, 43(3):1084-1093, 1997.

[7] K. Todros and J. Tabrikian, "Hybrid lower bound via compression of the sampled CLR function," Proc. of IEEE Workshop on SSP 2009.

[8] C. Ren, J. Galy, E. Chaumette, P. Larzabal, and A. Renaux, "Hybrid lower bound on the MSE based on the Barankin and Weiss-Weinstein bounds," in Proc. of IEEE ICASSP 2013.

[9] C. Ren, J. Galy, E. Chaumette, P. Larzabal, and A. Renaux, "A ZivZakaï type bound for hybrid parameter estimation," in Proc. of IEEE ICASSP 2014.

[10] L. Xu and X. Rong Li, "Hybrid Cramer-Rao Lower Bound on Tracking Ground Moving Extended Target," in Proc. of IEEE ISIF 2009.

[11] C. Ren, J. Galy, E. Chaumette, F. Vincent, P. Larzabal, A. Renaux, "Recursive hybrid Cramér-Rao bound for Discrete-Time Markovian Dynamic System", IEEE SP Letters, 22(10): 1543-1547, 2015.

[12] P. Tichavsky, C. Muravchik and A. Nehorai, "Posterior Cramér-Rao bounds for discrete-time nonlinear Filtering", IEEE Trans. on SP, 46(5):1386-1396, 1998. 